\title{
Weak Sequential Properties of the Multiplication Operators on Banach Algebras
}

\author{
Onur Oktay
}

July 15,2020

\begin{abstract}
Let $A$ be a Banach algebra. For $f \in A^{*}$, we inspect the weak sequential properties of the well-known map $T_{f}: A \rightarrow A^{*}, T_{f}(a)=f a$, where $f a \in A^{*}$ is defined by $f a(x)=f(a x)$ for all $x \in A$. We provide equivalent conditions for when $T_{f}$ is completely continuous for every $f \in A^{*}$, and for when $T_{f}$ maps weakly precompact sets onto L-sets for every $f \in A^{*}$. Our results have applications to the algebra of compact operators $K(X)$ on a Banach space $X$.
\end{abstract}

\section{Introduction}

It was conjectured in [12] that reflexive amenable Banach algebras are finite dimensional. Runde proved this conjecture with additional hypotheses. Precisely, every reflexive amenable Banach algebra $A$ with the approximation property, where the set of almost periodic functionals ap $(A)$ separates the points, is finite dimensional, see e.g., Corollary 3.3 and the preceding Remark in [20].

ap $(A)$ is related to the joint weak continuity properties of the multiplication [8]. When $A$ is reflexive, ap $(A)$ is separating if and only if the multiplication is jointly weakly sequentially continuous if and only if the multiplication operator $T_{f}$ is completely continuous for every $f \in A^{*}$. From this perspective, it is worthwhile to study the weak sequential properties of the multiplication and the associated operators for a general Banach algebra.

In Section 3, we study the joint weak sequential continuity of the Banach algebra multiplication, and the associated multiplication operators. In analogy to ap $(A)$, we define the subspaces $\operatorname{wpL}(A), \operatorname{lcc}(A)$ and $\operatorname{rcc}(A)$ of $A^{*}$. We provide characterizations of the joint weak sequential continuity of the multiplication in terms of those subspaces. We inspect the relationship between these functionals and several Banach space properties of $A$. The tools we develop in Section 3 have applications to the algebra of compact operators $K(X)$ on a Banach space $X$, which is contained in Section 4 . 


\section{Notation and Preliminaries}

Let $A$ be a Banach space. $A$ has Schur property if every weakly convergent sequence in $A$ is norm convergent. $A$ has Dunford-Pettis property (DPP) if $f_{n}\left(x_{n}\right) \rightarrow 0$ for all weakly null sequences $\left(x_{n}\right)$ in $A$ and $\left(f_{n}\right)$ in $A^{*}$. $A$ has Reciprocal Dunford-Pettis property (RDPP) if every completely continuous operator from $A$ into another Banach space is weakly compact.

$E \subseteq A^{*}$ is an $L$-set if for every weakly null sequences $\left(x_{n}\right)$ in $A$

$$
\lim _{n \rightarrow \infty} \sup _{f \in E}\left|f\left(x_{n}\right)\right|=0 .
$$

It is not difficult to show that $E$ is an L-set if and only if $f_{n}\left(x_{n}\right) \rightarrow 0$ for every sequence $\left(f_{n}\right)$ in $E$, and every weakly null sequence $\left(x_{n}\right)$ in $A$. Clearly, $A$ has Schur property if and only if the unit ball of $A^{*}$ is an L-set. $A$ has DPP if and only if every weakly precompact subset of $A^{*}$ is an L-set ([4], p.18). $A$ has RDPP if and only if every L-set contained in $A^{*}$ is relatively weakly compact [16],[11]. $A$ contains no copy of $\ell^{1}$ if and only if every L-set contained in $A^{*}$ is relatively compact [10].

When $A$ is a Banach algebra, one defines the left and right multiplication operators $L_{a}, R_{a}: A \rightarrow A$ by $L_{a} x=a x$ and $R_{a} x=x a$. Moreover,

$$
\begin{array}{lll}
f a(x)=f(a x) & \mu f(a)=\mu(f a) & \left(\mu \cdot \cdot_{1} \nu\right)(f)=\mu(\nu f) \\
a f(x)=f(x a) & f \mu(a)=\mu(a f) & (\nu \cdot 2 \mu)(f)=\mu(f \nu)
\end{array}
$$

for all $a, x \in A, f \in A^{*}$ and $\mu, \nu \in A^{* *}$. The products in the first column make $A^{*}$ a two-sided $A$-module, and naturally define two multiplication operators $S_{f}, T_{f}: A \rightarrow A^{*}$ given by $T_{f} a=f a$ and $S_{f} a=a f$, for each $f \in A^{*}$ and all $a \in A$. ap $(A)$ (resp. wap $(A)$ ) denotes the space of all $f \in A^{*}$ for which $T_{f}$ is a (weakly) compact operator. By duality, $T_{f}$ is (weakly) compact if and only if $S_{f}$ is (weakly) compact.

$A^{* *}$ is a Banach algebra with each of the Arens products $\cdot_{1}$ and $\cdot_{2}$. The canonical injection of $A$ into $A^{* *}$ is an isometric isomorphism onto its range for both products. $A$ is Arens regular if both Arens products are equal on $A^{* *}$. It is well-known that $A$ is Arens regular if and only if $A^{*}=\operatorname{wap}(A)$ [17]. Also $A^{*}=\operatorname{wap}(A)\left(\right.$ resp. $\left.A^{*}=\operatorname{ap}(A)\right)$ if and only if one (thus both) of the Arens products is separately (resp. jointly) bounded weak* continuous [8].

\section{Weak sequential continuity of multiplication}

Analogous to wap $(A)$ and $\operatorname{ap}(A)$, we define the subspaces wpL $(A), \operatorname{rcc}(A)$, $\operatorname{lcc}(A)$ and $\operatorname{cc}(A)$ as below.

Definition 3.1. Let $A$ be a Banach algebra.

i. $\operatorname{wpL}(A)$ is the set of all $f \in A^{*}$ such that $T_{f}$ maps weakly precompact sets onto L-sets, 
ii. lcc $(A)$ (resp. $\operatorname{rcc}(A))$ is the set of all $f \in A^{*}$ such that $T_{f}$ (resp. $S_{f}$ ) is completely continuous,

and $\operatorname{cc}(A)=\operatorname{lcc}(A) \cap \operatorname{rcc}(A)$.

Clearly $\operatorname{lcc}(A)=\operatorname{rcc}(A)$ if $A$ is commutative. Also ap $(A) \subseteq \operatorname{cc}(A)$, and $\operatorname{lcc}(A) \cup \operatorname{rcc}(A) \subseteq \operatorname{wpL}(A)$ by Lemmas 3.1 and 3.2 below.

Lemma 3.1. Let $A$ be a Banach algebra and $f \in A^{*}$. Then, the following are equivalent.

i. If $\left(x_{n}\right),\left(y_{n}\right)$ are weakly null sequences in $A$, then $f\left(x_{n} y_{n}\right) \rightarrow 0$.

ii. If $\left(x_{n}\right)$ is a weakly null and $\left(y_{n}\right)$ is a weakly Cauchy sequence in $A$, then $f\left(x_{n} y_{n}\right) \rightarrow 0$.

iii. $T_{f}$ map weakly precompact sets onto L-sets.

iii'. $S_{f}$ map weakly precompact sets onto L-sets.

Proof. $(i \Rightarrow i i)$ Suppose $f\left(x_{n} y_{n}\right) \not \rightarrow 0$. WLOG, there exist $r>0$ such that $\left|f\left(x_{n} y_{n}\right)\right| \geq r$ for all $n \in \mathbb{N}$. Since $\left(f x_{n}\right)$ is a weakly null sequence in $A^{*}$, then $f\left(x_{n_{k}} y_{k}\right) \rightarrow 0$ for a subsequence $\left(f x_{n_{k}}\right)_{k \in \mathbb{N}}$. Since $\left(y_{n_{k}}-y_{k}\right)_{k \in \mathbb{N}}$ is weakly null, then

$$
\lim _{k \rightarrow \infty} f\left(x_{n_{k}} y_{n_{k}}\right)=\lim _{k \rightarrow \infty} f\left(x_{n_{k}}\left(y_{n_{k}}-y_{k}\right)\right)+f\left(x_{n_{k}} y_{k}\right)=0 .
$$

Contradiction.

$\left(i i \Rightarrow\right.$ iii) Suppose there exists a weakly precompact $K \subseteq A$ such that $T_{f}(K)$ is not an L-set. Then, there exists a $r>0$ and a weakly null sequence $\left(y_{n}\right)$ in $A$ such that $\sup _{x \in K}\left|f x\left(y_{n}\right)\right| \geq 2 r$ for all $n \in \mathbb{N}$. Further, there exists a sequence $\left(x_{n}\right)$ in $K$ such that $\left|f\left(x_{n} y_{n}\right)\right| \geq r$ for all $n \in \mathbb{N}$. Since $K$ is weakly precompact, there is a weakly Cauchy subsequence $\left(x_{n_{k}}\right)$. Hence, $f\left(x_{n_{k}} y_{n_{k}}\right) \nrightarrow \rightarrow 0$ while $\left(x_{n_{k}}\right)$ is weakly Cauchy and $\left(y_{n_{k}}\right)$ weakly null. Contradiction.

Assuming that $S_{f}$ does not map weakly precompact sets onto L-sets similarly leads to a contradiction.

$($ iii $\Rightarrow i)$ Let $\left(x_{n}\right),\left(y_{n}\right)$ be weakly null. $\left\{f x_{n}: n \in \mathbb{N}\right\}$ is an L-set by hypothesis, so $f\left(x_{n} y_{n}\right) \rightarrow 0$.

Lemma 3.2. Let $A$ be a Banach algebra and $f \in A^{*}$. Then, the following are equivalent.

i. If $\left(x_{n}\right)$ is weakly null and $\left(y_{n}\right)$ is bounded, then $f\left(x_{n} y_{n}\right) \rightarrow 0$ (resp. $\left.f\left(y_{n} x_{n}\right) \rightarrow 0\right)$

ii. $T_{f}\left(\right.$ resp. $\left.S_{f}\right)$ is completely continuous.

iii. $S_{f}\left(\operatorname{resp} . T_{f}\right)$ maps bounded sets onto L-sets. 
Proof. $(i \Rightarrow i i)$ Suppose $T_{f}$ is not completely continuous. Then, there exists a weakly null $\left(x_{n}\right)$ and $r>0$ such that $\left\|f x_{n}\right\| \geq 2 r$ for all $n \in \mathbb{N}$. For each $n \in \mathbb{N}$, there exists $y_{n} \in A,\left\|y_{n}\right\|=1$ such that $\left|f\left(x_{n} y_{n}\right)\right| \geq r$. Hence, $f\left(x_{n} y_{n}\right) \nrightarrow \rightarrow 0$.

$(i i \Rightarrow i)$ Since $\left\|f x_{n}\right\| \rightarrow 0$, then $\left|f\left(x_{n} y_{n}\right)\right| \leq\left\|f x_{n}\right\|\left\|y_{n}\right\| \rightarrow 0$.

$\left(i \Rightarrow\right.$ iii) Suppose $S_{f}$ does not map bounded sets onto L-sets. Then, there exists a bounded $\left(y_{n}\right)_{n \in \mathbb{N}}$, a weakly null $\left(x_{n}\right)$ and $r>0$ such that $\left|y_{n} f\left(x_{n}\right)\right| \geq r$ for all $n \in \mathbb{N}$. Thus, $f\left(x_{n} y_{n}\right) \not \rightarrow 0$.

$(i i i \Rightarrow i)$ Since $\left\{y_{n} f: n \in \mathbb{N}\right\}$ is an L-set by hypothesis, then $f\left(x_{n} y_{n}\right)=y_{n} f\left(x_{n}\right) \rightarrow 0$.

Lemma 3.3. Let $A$ be a Banach algebra and $f \in \operatorname{wpL}(A)$. The following are equivalent.

i. $f \notin \operatorname{lcc}(A)$ (resp. $f \notin \operatorname{rcc}(A))$.

ii. $A$ contains an $\ell^{1}$ sequence $\left(w_{n}\right)$ and a weakly null sequence $\left(u_{n}\right)$ such that $f\left(u_{m} w_{n}\right)=\delta_{m n}\left(\right.$ resp. $\left.f\left(w_{n} u_{m}\right)=\delta_{m n}\right)$ for all $m \geq n$.

Proof. (ii $\Rightarrow$ i) Clear, since $E=\left\{w_{n}: n \in \mathbb{N}\right\}$ is a bounded set such that $T_{f}(E)=\left\{f w_{n}: n \in \mathbb{N}\right\}$ (resp. $\left.S_{f}(E)=\left\{w_{n} f: n \in \mathbb{N}\right\}\right)$ is not an L-set. Thus, $f \notin \operatorname{lcc}(A)$ (resp. $f \notin \operatorname{rcc}(A))$.

$(i \Rightarrow$ ii) If $f \notin \operatorname{lcc}(A)$ (resp. $f \notin \operatorname{rcc}(A))$, then there exist $r>0$, a weakly null sequence $\left(x_{n}\right)$ and a bounded sequence $\left(y_{n}\right)$ such that $\left|f\left(x_{n} y_{n}\right)\right| \geq r$ (resp. $\left.\left|f\left(y_{n} x_{n}\right)\right| \geq r\right)$ by Lemma 3.2. $\left(y_{n}\right)$ cannot have a weakly Cauchy subsequence by Lemma 3.1, thus it has an $\ell^{1}$ subsequence by Rosenthal's theorem. Without loss of generality, we may assume $\left(y_{n}\right)$ is an $\ell^{1}$ sequence. Further, replacing $y_{n}$ by $\tilde{y}_{n}=y_{n} /\left\|y_{n}\right\|$ and $x_{n}$ by $\tilde{x}_{n}=\left|f\left(x_{n} y_{n}\right)\right|^{-1}\left\|y_{n}\right\| x_{n}$ (resp. $\left.\quad \tilde{x}_{n}=\left|f\left(x_{n} y_{n}\right)\right|^{-1}\left\|y_{n}\right\| x_{n}\right)$, we may assume that $f\left(x_{n} y_{n}\right)=1$ (resp. $\left.f\left(y_{n} x_{n}\right)=1\right)$ for a weakly null sequence $\left(x_{n}\right)$ and a unit norm $\ell^{1}$ sequence $\left(y_{n}\right)$.

First, assume $f \notin \operatorname{lcc}(A)$ and $\|f\| \leq 1$. Let $c>0$ be a bound for the sequence $\left(\left\|x_{n}\right\|\right)$. Proof is by induction. For the base step, let $u_{1}=x_{1}$ and $w_{1}=y_{1}$. For the inductive step, assume that, for $k, l=1, \ldots, n-1$, there exist $x_{m_{k}}, u_{k}$ such that $\left\|x_{m_{k}}-u_{k}\right\| \leq(1+c)^{-k}, f\left(u_{l} y_{n_{k}}\right)=\delta_{k l}$ for $l \geq k$. Here, $w_{k}=y_{m_{k}}$.

We will construct $u_{n}$ and $w_{n}$. Since $\left(x_{n}\right)$ is weakly null, there exists $m_{n} \geq$ $m_{n-1}$ such that

$$
\max \left\{\left|f\left(x_{m_{n}} w_{k}\right)\right|: k=1, \ldots, n-1\right\} \leq \frac{(1+c)^{-(n+2)}}{1+(1+c)^{-(n+1)}} .
$$

There exists $\tilde{u}_{n} \in \bigcap_{k=1}^{n-1} \operatorname{ker}\left(w_{k} f\right)$ such that

$$
\left\|x_{m_{n}}-\tilde{u}_{n}\right\| \leq \frac{(1+c)^{-(n+1)}}{1+(1+c)^{-(n+1)}} .
$$

Let $w_{n}=y_{m_{n}}$ and $u_{n}=\tilde{u}_{n} / f\left(\tilde{u}_{n} w_{n}\right)$. Then, $f\left(u_{n} w_{n}\right)=1, f\left(u_{n} w_{k}\right)=0$ for $k=1, \ldots, n-1$, and

$$
\left\|x_{m_{n}}-u_{n}\right\| \leq\left\|x_{m_{n}}-\tilde{u}_{n}\right\|+\left\|\tilde{u}_{n}\right\| \frac{\left|1-f\left(\tilde{u}_{n} w_{n}\right)\right|}{\left|f\left(\tilde{u}_{n} w_{n}\right)\right|} \leq(1+c) \frac{\left\|x_{m_{n}}-\tilde{u}_{n}\right\|}{1-\left\|x_{m_{n}}-\tilde{u}_{n}\right\|} \leq(1+c)^{-n} .
$$


Consequently, $\left(u_{n}\right)$ is weakly null. Clearly, being a subsequence of an $\ell^{1}$ sequence, $\left(w_{n}\right)$ is an $\ell^{1}$ sequence.

The other case, $f \notin \operatorname{rcc}(A)$, is handled similarly.

Remark. If not only the sequence $\left(u_{n}\right)$ in Lemma 3.3 is weakly null, but also $\sum u_{n}$ is a weakly unconditionally convergent series, then $\left(w_{n}\right)$ is a complemented $\ell^{1}$ sequence. In fact, let $W$ be the closed linear span of $\left(w_{n}\right)$, which is isomorphic to $\ell^{1}$. Let $S: A \rightarrow W$ be defined by $S x=\sum_{n \in \mathbb{N}} f\left(u_{n} x\right) w_{n}$ (resp. $S x=$ $\left.\sum_{n \in \mathbb{N}} f\left(x u_{n}\right) w_{n}\right)$. Then, $S$ is a surjective bounded linear operator, and thus, $A$ contains a complemented copy of $\ell^{1}$, e.g., by Theorem 5 in page 72 of [5].

Lemma 3.4. Let $A$ be a Banach algebra, $f \in A^{*}$ and $a, b \in A$.

a. If $f \in \operatorname{wpL}(A)$ and $L_{a}$ (resp. $\left.R_{a}\right)$ is a weakly precompact operator, then $f a \in \operatorname{rcc}(A)($ resp. af $\in \operatorname{lcc}(A))$.

b. If $f \in \operatorname{lcc}(A)$ (resp. $f \in \operatorname{rcc}(A))$ and $L_{a}$ (resp. $R_{a}$ ) is weakly precompact, then $f a \in \operatorname{ap}(A)$ (resp. af $\in$ ap $(A)$ ).

c. If $f \in \mathrm{wpL}(A)$ and $L_{a}, R_{b}$ are weakly precompact operators, then $b f a \in$ ap $(A)$.

Proof. First, $T_{f a}=T_{f} L_{a}$ and $S_{a f}=S_{f} R_{a}$.

a. If $B$ is a bounded subset, then $T_{f a}(B)=T_{f}\left(L_{a}(B)\right)$ is an L-set. Thus, $f a \in \operatorname{rcc}(A)$ by Lemma 3.2. Similarly, $S_{a f}$ maps bounded sets onto L-sets, so af $\in \operatorname{lcc}(A)$ by Lemma 3.2.

b. If $B$ is a bounded subset, then $T_{f a}(B)=T_{f}\left(L_{a}(B)\right)$ is relatively compact, so $f a \in$ ap $(A)$. Similarly, $S_{a f}=S_{f} R_{a}$ is a compact operator, i.e., af $\in \operatorname{ap}(A)$.

c. a direct consequence of (a) and (b).

Theorem 3.5. Let $A$ be a Banach algebra. If $A$ contains no copy of $\ell^{1}$, then $\operatorname{wpL}(A)=\operatorname{cc}(A)=\operatorname{ap}(A)$.

Proof. $\operatorname{wpL}(A)=\operatorname{cc}(A)$ by Lemma 3.3. Second, let $f \in \operatorname{cc}(A)$. Every completely continuous operator from $A$ is compact when $A$ does not contain a copy of $\ell^{1}$. Thus, $T_{f}$ is compact, i.e., $f \in \operatorname{ap}(A)$.

Definition 3.2. Let $A$ be a Banach algebra. The multiplication of $A$ is jointly weakly sequentially continuous (jwsc) if whenever $\left(x_{n}\right),\left(y_{n}\right)$ are weakly null sequences, then so is $\left(x_{n} y_{n}\right)$.

We say that the multiplication is $l$-strong jwsc (resp. $r$-strong jwsc) if $\left(x_{n} y_{n}\right)$ (resp. $\left.\left(y_{n} x_{n}\right)\right)$ is weakly null whenever $\left(x_{n}\right)$ is a weakly null sequence, and $\left(y_{n}\right)$ is a bounded sequence. The multiplication is strong jwsc if it is both l-strong and r-strong jwsc.

The space of continuous functions $C(K)$ on a compact Hausdorff topological space is an example of a Banach algebra with strong jwsc multiplication. The group algebra $L^{1}(\mathbb{R})$ is an example with jwsc multiplication, which is neither l-strong jwsc nor r-strong jwsc. 
Theorem 3.6. Let $A$ be a Banach algebra. Then,

a. $A^{*}=\operatorname{wpL}(A)$ if and only if $A$ has jwsc multiplication.

b. $A^{*}=\operatorname{lcc}(A)$ (resp. $\left.A^{*}=\operatorname{rcc}(A)\right)$ if and only if $A$ has l-strong (resp. r-strong) jwsc multiplication.

c. If $A$ has $\mathrm{DPP}$, then $A^{*}=\mathrm{wpL}(A)$. If $A$ is also Arens regular, then $A^{*}=\operatorname{cc}(A)$.

d. If $A$ contains no copy of $\ell^{1}$ and has jwsc multiplication, then $A^{*}=\operatorname{ap}(A)$.

e. If $A^{*}$ has Schur property, then $A^{*}=\operatorname{ap}(A)$.

f. If $A$ has Schur property, then $A^{*}=\operatorname{cc}(A)$.

Proof. a. Immediate by Lemma 3.1. b. Immediate by Lemma 3.2.

c. It is well-known that if $A$ has DPP, then $A$ has jwsc multiplication, see e.g., Proposition 2.34 in [7]. In fact, let $f \in A^{*}$ and $\left(x_{n}\right),\left(y_{n}\right)$ be two weakly null sequences in $A$. Since $\left(f x_{n}\right)$ is a weakly null sequence in $A^{*}$, then $f\left(x_{n} y_{n}\right)=f x_{n}\left(y_{n}\right) \rightarrow 0$ by the DPP. Thus, $A^{*}=\operatorname{wpL}(A)$ by (a).

Second, $A^{*}=\operatorname{wap}(A)$ by Arens regularity and wap $(A) \subseteq \operatorname{cc}(A)$ by DPP. Thus, $A^{*}=\operatorname{cc}(A)$.

d. By (a) and Theorem 3.5.

e. $A^{*}$ has Schur property if and only if $A$ has DPP and contains no copy of $\ell^{1}$ (see Theorem 3 in [4]). Thus, ap $(A)=A^{*}$ by (c) and (d).

f. Clearly, every bounded linear operator defined on $A$ is completely continuous.

It is worth to note that there are commutative Banach algebras, which have DPP, but $A^{*} \neq \operatorname{cc}(A)$. In fact, if $G$ is a non-discrete non-compact locally compact Abelian group, then the group algebra $L^{1}(G)$ has the DPP, and there exists $g \in C_{b}(G)$ for which the map $T_{g}: L^{1}(G) \rightarrow L^{\infty}(G), T_{g}(x)=g * x$ is not completely continuous [3].

On the other hand, there are commutative Banach algebras, which does not have DPP, but $A^{*}=\operatorname{ap}(A)$. In fact, $\ell^{p}(1<p<\infty)$ is a Banach algebra with pointwise operations. It is not difficult to show that the pointwise multiplication is jwsc. Being reflexive, $\ell^{p}$ does not have DPP and contains no copy of $\ell^{1}$. Thus, $A^{*}=\operatorname{ap}(A)$ by $(\mathrm{d})$ of Theorem 3.6.

It is clear that (e) of Theorem 3.6 does not have a converse for $\ell^{p}$. However, if $A$ is a $\mathrm{C}^{*}$-algebra, then $A^{*}=\operatorname{ap}(A)$ if and only if $A^{*}$ have Schur property by Theorem 3.6 in [15]. We provide an analogous statement below.

Corollary 3.7. Let $A$ be a $\mathrm{C}^{*}$-algebra. Then, the following are equivalent.

i. $A^{*}=\operatorname{cc}(A)$.

ii. $A^{*}=\operatorname{wpL}(A)$.

iii. $A$ has DPP. 
Proof. $(i \Rightarrow i i)$ clear since cc $(A) \subseteq \mathrm{wpL}(A)$.

(ii $\Rightarrow$ iii) if $A^{*}=\operatorname{wpL}(A)$, then $A$ has jwsc multiplication by Theorem 3.6.

Particularly, $\left(x_{n} x_{n}^{*}\right)$ is a weakly null sequence whenever $\left(x_{n}\right)$ is weakly null.

Equivalently, $A$ has DPP by Theorem 1 in [2].

$(i i i \Rightarrow i) \mathrm{C}^{*}$-algebras are Arens regular, so $A^{*}=\operatorname{wap}(A)$. Every weakly compact operator defined on $A$ is completely continuous by DPP. Thus, wap $(A) \subseteq$ $\operatorname{cc}(A)$ and so $A^{*}=\operatorname{cc}(A)$.

Theorem 3.8. Let $A$ be a Banach algebra, which is a left (resp. right) ideal in $A^{* *}$. If the multiplication is jwsc, then

a. $A^{*} A \subseteq \operatorname{rcc}(A)\left(\operatorname{resp} . A A^{*} \subseteq \operatorname{lcc}(A)\right)$.

b. $A A^{*} A \subseteq$ ap $(A)$.

Proof. It is well-known that $A$ is a left (resp. right) ideal in $A^{* *}$ if and only if $L_{a}$ (resp. $R_{a}$ ) is weakly compact for all $a \in A$, see e.g., [17]. Second, $A^{*}=\operatorname{wpL}(A)$ by Theorem 3.6.

a. $f a \in \operatorname{rcc}(A)$ (resp. $a f \in \operatorname{lcc}(A))$ for all $f \in A^{*}$ and $a \in A$ by Lemma 3.4. Hence, $A^{*} A \subseteq \operatorname{rcc}(A)$ (resp. $A A^{*} \subseteq \operatorname{lcc}(A)$ ).

b. bfa $\in$ ap $(A)$ for all $f \in A^{*}$ and $a, b \in A$ by Lemma 3.4. Hence, the result.

It is clear from the proof that the result of Theorem 3.8 persists if we assumed that $L_{a}$ (resp. $R_{a}$ ) is weakly precompact for all $a \in A$, instead of $A A^{* *} \subseteq A$ (resp. $A^{* *} A \subseteq A$ ). The same is valid for the next corollary.

Corollary 3.9. Let $A$ be a Banach algebra with a bounded left (resp. right) approximate identity, which is a right (resp. left) ideal in $A^{* *}$. If the multiplication of $A$ is jwsc, then $\operatorname{wap}(A) \subseteq \operatorname{lcc}(A)(\operatorname{resp} . \quad w a p(A) \subseteq \operatorname{rcc}(A))$. If, additionally, $A$ is Arens regular, then $A^{*}=\operatorname{lcc}(A)\left(\operatorname{resp} . A^{*}=\operatorname{rcc}(A)\right)$.

Proof. wap $(A) \subseteq A A^{*}$ (resp. wap $(A) \subseteq A^{*} A$ ) by Theorem 3.1 in [21]. Thus, if the multiplication is jwsc, then $\operatorname{wap}(A) \subseteq \operatorname{lcc}(A)(\operatorname{resp} . \operatorname{wap}(A) \subseteq \operatorname{rcc}(A))$ by Theorem 3.8. The second result is obvious since $A^{*}=\operatorname{wap}(A)$ if $A$ is Arens regular.

\section{The Algebra of Compact Operators $K(X)$}

Let $X$ be a Banach space. $K(X)$ denotes the Banach algebra of compact operators $X \rightarrow X$. The following theorem summarizes some of the well-known results about $K(X)$, which we will need subsequently.

Theorem 4.1. Suppose $X$ is an infinite dimensional Banach space and let $A=K(X)$. Then,

a. $\operatorname{wap}(A)=A^{*}$ if and only if $X$ is reflexive.

b. $\operatorname{wpL}(A)=A^{*}$ if and only if $X$ has DPP. 
c. If $X$ has the approximation property, then $\operatorname{ap}(A)=\{0\}$.

d. A cannot both be Arens regular and have jwsc multiplication.

e. If $X$ is reflexive, then $A$ contains no copy of $\ell^{1}$.

f. If $\operatorname{wpL}(A)=A^{*}$, then $A$ contains a copy of $\ell^{1}$.

Proof. a. Theorem 3 in [22].

b. [1] and Theorem 3.6.

c. Proposition 3.3 in [9].

d. by (a.), (b.), and the fact that every reflexive Banach space with the DPP is finite dimensional.

e. $A^{*}$ has Radon-Nikodym property by [19]. Equivalently, every separable subspace of $A$ has a separable dual [6, p.198]. Hence, $A$ cannot contain a copy of $\ell^{1}$.

f. $X$ has DPP by (b.). Thus, either $X$ contains a copy of $\ell^{1}$, or $X^{*}$ has Schur property. Also, every infinite dimensional Banach space with Schur property contains a copy of $\ell^{1}$. Hence, either $X$ or $X^{*}$, both of which are isometrically isomorphic to complemented subspaces of $A$, contains a copy of $\ell^{1}$.

Theorem 4.2. Let $X$ be an infinite dimensional Banach space with the approximation property and $A=K(X)$.

a. If $\operatorname{wpL}(A)$ separates the points of $A$, then either $L_{a}$ fixes a copy of $\ell^{1}$ for every nonzero $a \in A$, or $R_{a}$ fixes a copy of $\ell^{1}$ for every nonzero $a \in A$.

b. If lcc $(A)($ resp. $\operatorname{rcc}(A))$ separates the points of $A$, then $L_{a}$ (resp. $R_{a}$ ) fixes a copy of $\ell^{1}$ for every nonzero $a \in A$.

Proof. A bounded linear operator between two Banach spaces is weakly precompact if and only if it does not fix a copy of $\ell^{1}$.

a. Assume, for a contradiction, that $L_{a}$ and $R_{b}$ are weakly precompact operators for two nonzero $a, b \in A$. Then, $b f a \in \operatorname{ap}(A)$ for all $f \in \operatorname{wpL}(A)$ by Lemma 3.4. But, $\operatorname{ap}(A)=\{0\}$ by Theorem 4.1.c. Thus, $b f a=0$ for all $f \in \operatorname{wpL}(A)$. Since $\operatorname{wpL}(A)$ is separating, then $L_{a} R_{b}=0$. Hence, either $a=0$ or $b=0$. Contradiction.

b. If $R_{a}$ (resp. $L_{a}$ ) is weakly precompact, then af $\in \operatorname{ap}(A)$ (resp. $f a \in$ ap $(A))$ for all $f \in \operatorname{rcc}(A)$ (resp. $f \in \operatorname{lcc}(A)$ ). Since ap $(A)=\{0\}$ and $\operatorname{rcc}(A)$ (resp. lcc $(A)$ ) is a separating set, then $R_{a}=0$ (resp. $L_{a}=0$ ) so $a=0$.

Theorem 4.3 is analogous to Asthagiri's theorem in [1]. Perhaps known by the experts in the field, Theorem 4.3 never appeared in the literature to the best of our knowledge. The proof is almost verbatim similar to Asthagiri's proof in [1], nonetheless the key differences are not entirely obvious. Thus, we provide a separate proof beolow.

Theorem 4.3. Let $X$ be a Banach space and $A=K(X)$.

a. $A^{*}=\operatorname{rcc}(A)$ if and only if $X$ has Schur property. 
b. $A^{*}=\operatorname{lcc}(A)$ if and only if $X^{*}$ has Schur property.

c. $A^{*} \neq \operatorname{cc}(A)$ for any infinite dimensional Banach space $X$.

Proof. a. First assume $A^{*}=\operatorname{rcc}(A)$. Let $\left(x_{n}\right)$ be a weakly null sequence in $X$. It is sufficient to show that $f_{n}\left(x_{n}\right) \rightarrow 0$ for any bounded sequence $\left(f_{n}\right)$ in $X^{*}$.

$\left(x_{1} \otimes f_{n}\right)$ is a bounded, $\left(x_{n} \otimes f_{1}\right)$ is a weakly null sequence in $K(X)$. Thus, $f_{n}\left(x_{n}\right) x_{1} \otimes f_{1}=\left(x_{1} \otimes f_{n}\right)\left(x_{n} \otimes f_{1}\right) \rightarrow 0$ weakly in $K(X)$. Hence, $f_{n}\left(x_{n}\right) \rightarrow 0$.

Conversely, suppose $\left(x_{n}\right)$ is a bounded and $\left(y_{n}\right)$ is a weakly null sequence in $K(X)$. For each $\nu \in X^{* *},\left(y_{n}^{* *} \nu\right)$ is a weak* null sequence in $X^{* *}$ by [14]. On the other hand, $y_{n}^{* *} \nu \in X$ since $y_{n}$ are compact. Thus, $\left(y_{n}^{* *} \nu\right)$ is weakly null in $X$, and so norm null by the Schur property.

Hence, for each $f \in X^{*}$ and $\nu \in X^{* *}$,

$$
\left|\nu\left(\left(x_{n} y_{n}\right)^{*} f\right)\right|=\left|y_{n}^{* *} \nu\left(x_{n}^{*} f\right)\right| \leq\left\|y_{n}^{* *} \nu\right\|\left\|x_{n}^{*} f\right\| \rightarrow 0,
$$

i.e., $x_{n} y_{n} \rightarrow 0$ weakly in $K(X)$. Thus, $A^{*}=\operatorname{rcc}(A)$ by Lemma 3.2 .

b. Assume $A^{*}=\operatorname{lcc}(A)$ and let $\left(f_{n}\right)$ be a weakly null sequence in $X^{*}$. It is sufficient to show that $f_{n}\left(x_{n}\right) \rightarrow 0$ for any bounded sequence $\left(x_{n}\right)$ in $X$.

$\left(x_{1} \otimes f_{n}\right)$ is a weakly null, $\left(x_{n} \otimes f_{1}\right)$ is a bounded sequence in $K(X)$. Thus, $f_{n}\left(x_{n}\right) x_{1} \otimes f_{1}=\left(x_{1} \otimes f_{n}\right)\left(x_{n} \otimes f_{1}\right) \rightarrow 0$ weakly in $K(X)$. Hence, $f_{n}\left(x_{n}\right) \rightarrow 0$.

Conversely, suppose $\left(x_{n}\right)$ is a weakly null and $\left(y_{n}\right)$ is a bounded sequence in $K(X)$. For each $f \in X^{*},\left(x_{n}^{*} f\right)$ is weakly null in $X^{*}$ by [14], and so norm null by the Schur property. Hence, for each $f \in X^{*}$ and $\nu \in X^{* *}$,

$$
\left|\nu\left(\left(x_{n} y_{n}\right)^{*} f\right)\right|=\left|y_{n}^{* *} \nu\left(x_{n}^{*} f\right)\right| \leq\left\|y_{n}^{* *} \nu\right\|\left\|x_{n}^{*} f\right\| \rightarrow 0,
$$

i.e., $x_{n} y_{n} \rightarrow 0$ weakly in $K(X)$. Thus, $A^{*}=\operatorname{lcc}(A)$ by Lemma 3.2.

c. $X$ and $X^{*}$ cannot both have Schur property unless $X$ is finite dimensional.

An immediate consequence of Theorem 4.3 is given below.

Corollary 4.4. Let $X$ be an infinite dimensional Banach space with the approximation property and $A=K(X)$.

a. If $X$ has DPP, then either $L_{a}$ fixes a copy of $\ell^{1}$ for every nonzero $a \in A$, or $R_{a}$ fixes a copy of $\ell^{1}$ for every nonzero $a \in A$.

b. If $X$ (resp. $X^{*}$ ) has Schur property, then $R_{a}$ (resp. $L_{a}$ ) fixes a copy of $\ell^{1}$ for every nonzero $a \in A$.

Proof. a. by Theorems 4.2 and 4.1.b. $\quad$ b. by Theorems 4.2 and 4.3 .

\section{References}

[1] R.K. Asthagiri, A new characterization of the Dunford-Pettis property, Proceedings of the Royal Irish Academy. Section A: Mathematical and Physical Sciences 83A (1983), no. 2, 169-170. 
[2] C.-H. Chu and B. Iochum, The Dunford-Pettis property in $C^{*}$-algebras, Studia Mathematica 97 (1990), no. 1, 59-64.

[3] G. Crombez and W. Govaerts, Completely continuous multipliers from $L^{1}(G)$ into $L^{\infty}(G)$, Ann. Inst. Fourier (Grenoble) 34 (1984), no. 2, 137154 .

[4] J. Diestel, A survey of results related to the Dunford-Pettis property, Contemporary Mathematics, vol. 2, pp. 15-60, American Mathematical Society, 1980 .

[5] _ Sequences and Series in Banach Spaces, Graduate Texts in Mathematics, Springer, 1984.

[6] J. Diestel and J.J. Uhl, Vector Measures, Mathematical Surveys, vol. 15, American Mathematical Society, Providence RI, 1977.

[7] S. Dineen, Complex Analysis on Infinite Dimensional Spaces, Monographs in Mathematics, Springer, 1999.

[8] J. Duncan and S.A.R. Hosseiniun, The second dual of a Banach algebra, Proceedings of the Royal Society of Edinburgh 84A (1979), 309-325.

[9] J. Duncan and A. Ülger, Almost periodic functionals on Banach algebras, The Rocky Mountain Journal of Mathematics 22 (1992), no. 3, 837-848.

[10] G. Emmanuele, A dual characterization of Banach spaces not containing $\ell^{1}$, Bulletin of the Polish Academy of Sciences, Mathematics 34 (1986), no. $3-4,155-160$.

[11] G. Emmanuele, On the reciprocal Dunford-Pettis property in projective tensor products, Mathematical Proceedings of the Cambridge Philosophical Society 109 (1991), 161-166.

[12] J.E. Gale, T.J. Ransford, and M.C. White, Weakly compact homomorphisms, Transactions of the American Mathematical Society 331 (1992), no. $2,815-824$.

[13] G. Godefroy and B. Iochum, Arens-regularity of Banach algebras and the geometry of Banach spaces, Journal of Functional Analysis 80 (1988), no. 1, $47-59$.

[14] N.J. Kalton, Spaces of compact operators, Mathematische Annalen 208 (1974), no. 4, 267-278.

[15] A. To-Ming Lau and A. Ülger, Some geometric properties on the Fourier and Fourier-Stieltjes algebras of locally compact groups, Arens regularity and related problems, Transactions of the American Mathematical Society (1993), 321-359. 
[16] T. Leavelle, The reciprocal Dunford-Pettis and Radon-Nikodym properties in Banach spaces, Ph.D. thesis, University of North Texas, 1984.

[17] T.W. Palmer, Banach Algebras and the General Theory of *-Algebras I, Cambridge University Press, 1994.

[18] H. Pfitzner, Weak compactness in the dual of a $C^{*}$-algebra is determined commutatively, Mathematische Annalen 298 (1994), no. 2, 349-372.

[19] W. Ruess, Duality and geometry of spaces of compact operators, NorthHolland Mathematics Studies (K.D. Bierstedt and B. Fuchssteiner, eds.), Functional Analysis: Surveys and Recent Results III, vol. 90, NorthHolland, 1984, pp. 59-78.

[20] V. Runde, Amenability for dual Banach algebras, Studia Mathematica 148 (2001), 47-66.

[21] A. Ülger, Arens regularity sometimes implies the RNP, Pacific Journal of Mathematics 143 (1990), no. 2, 377-399.

[22] N.J. Young, Periodicity of functionals and representations of normed algebras on reflexive spaces, Proceedings of the Edinburgh Mathematical Society 20 (1976), no. 2, 99-120. 